\title{
Why spin ice obeys the ice rules
}

\author{
S. V. Isakov, ${ }^{1}$ R. Moessner, ${ }^{2}$ and S. L. Sondhi ${ }^{3}$ \\ ${ }^{1}$ Department of Physics, University of Toronto, Toronto, Ontario, Canada M5S $1 A 7$ \\ ${ }^{2}$ Laboratoire de Physique Théorique de l'Ecole Normale Supérieure, CNRS-UMR8549, Paris, France and \\ ${ }^{3}$ Department of Physics, Princeton University, Princeton, NJ, USA
}

\begin{abstract}
The low temperature entropy of the the spin ice compounds, such as $\mathrm{Ho}_{2} \mathrm{Ti}_{2} \mathrm{O}_{7}$ and $\mathrm{Dy}_{2} \mathrm{Ti}_{2} \mathrm{O}_{7}$, is well described by the nearest-neighbor antiferromagnetic Ising model on the pyrochlore lattice, i.e. by the "ice rules". This is surprising since the dominant coupling between the spins is their long ranged dipole interaction. We show that this phenomenon can be understood rather elegantly: one can construct a model dipole interaction, by adding terms of shorter range, which yields precisely the same ground states, and hence $T=0$ entropy, as the nearest neighbor interaction. A treatment of the small difference between the model and true dipole interactions reproduces the numerical work by Gingras et al. in detail. We are also led to a more general concept of projective equivalence between interactions.
\end{abstract}

PACS numbers: 75.10.Hk, 75.50.Ee, 75.40.Cx

Introduction: In 1956, Anderson [1] observed that an Ising antiferromagnet on the pyrochlore lattice would exhibit a macroscopic ground state entropy equivalent to that of water ice 2]. Almost four decades later, in 1997, such "spin ice" behavior was experimentally discovered. Harris et al. noticed that the pyrochlore compound $\mathrm{Ho}_{2} \mathrm{Ti}_{2} \mathrm{O}_{7}$, where only the Holmium ions are magnetic, failed to display any signs of ordering down to below the ferromagnetic Curie temperature deduced from its high temperature susceptibility. They proposed that this was due to the presence of an easy-axis anisotropy, which leads to the ferromagnet effectively becoming an Ising pseudospin antiferromagnet at temperatures well below the anisotropy strength [3]. This interpretation was strongly supported by a remarkable experiment, in which Ramirez et al. showed that the related compound $\mathrm{Dy}_{2} \mathrm{Ti}_{2} \mathrm{O}_{7}$ displayed a residual entropy, $\mathcal{S}_{0}$, at low temperatures, which was in excellent agreement with the Pauling estimate for water ice, $\mathcal{S}_{0} \approx(1 / 2) \ln (3 / 2)[4]$.

However, it was quickly pointed out that the dominant interaction in these compounds is dipolar due to the large moments on $\mathrm{Ho}^{3+}$ and $\mathrm{Dy}^{3+}, \mu \approx 10 \mu_{B}$, where $\mu_{B}$ is the Bohr magneton [5]. Yet, the dipole interaction is long ranged, decaying with separation, $r$, as $1 / r^{3}$. Why, then, is the entropy of the nearest-neighbor model stable to the inclusion of the rest of this interaction $[5,6]$ ?

That this is the case has been checked in detail by explicit simulation. Further, two qualitative observations have been made in the literature. First, the anisotropic nature of the dipole interaction precludes any obvious ordering instability stemming from its long range. It is probably fair to say that it is now agreed that instead, it leads to a weak ordering instability away from $\mathbf{q}=0$ $[5,6,7,8]$, albeit one which is not observed [9]. Second, explicit evaluation of the Fourier transform of the easy-axis projected dipole interaction to large (and, using Ewald summation, infinite) distances yielded a surprise: the infinite distance result resembled the nearest- neighbor interaction more than an interaction truncated at, say, 10 or so nearest neighbors; this 'self-screening' (Gingras) indicates that there is something special about the dipole interaction [10, 11].

In this paper we show what it is that is special about spin ice and dipole interactions. The main insight comes from recent progress in understanding the correlations dictated by the ice rule 12, 13, 14, 15]: it was shown that the pyrochlore Ising model dynamically acquires a gauge structure at $T=0$, which manifests itself in the emergence of dipolar correlations as the ice rules are enforced. The crucial observation is that this gauge structure is (formally, not in origin) exactly the same as that of 'ordinary' magnetostatics; this latter, of course, is what determines the form of the dipole interactions.

Most crisply, we use this observation to show that there exists a slightly modified "model" dipole interaction, which differs from the physical interaction by terms that fall off faster and are small in magnitude, whose ground states are identically the same as those of the nearest neighbor interaction. This accounts for their identical low temperature entropy.

The mathematical implementation of this insight revolves around relating two quantities - dipole interactions, and the operator enforcing the ice rules - to the same projector, $\mathcal{P}$. Finally, this leads us to a generalizable equivalence between a long ranged interaction and one of shorter range projected onto its low energy manifold; this we term "projective equivalence".

In the balance of the paper we flesh out these statements. We begin by recalling the energetics of spin ice. The ground state degeneracy of the nearest-neighbor antiferromagnet manifests itself in a pair of flat bands. We show next that a model dipole interaction possessing the correct long distance form, leads to exactly the same pair of flat bands and thence to the same ground state manifold, even with arbitrary admixture of the nearestneighbor interaction (as long as not so overwhelmingly 
antiferromagnetic to invert the ordering of the bands). The deviation of the model interaction from the true dipole interaction is seen to vanish as an integrable $r^{-5}$. Treating this by elementary means we are able to account for the weak residual dispersion and ordering tendency found in previous work. As a byproduct, this analysis makes clear that at higher temperatures, the correlations arising from the dipole interaction are not the same as those stemming from the ice rules alone. We close with some comments on projective equivalence.

Hamiltonian: The dominant term in the spin ice Hamiltonian is a strong easy easy axis anisotropy, which allows us to transform the vector spins $\mathbf{S}$ to Ising pseudospins $\sigma= \pm 1$ via $\mathbf{S}_{i \alpha}=\sigma_{i \alpha} \mathbf{e}_{\alpha}$ (no sum over $\alpha$ ). $\mathbf{e}_{\alpha}$ denote the local easy axes of the pyrochlore lattice, which consists of corner-sharing tetrahedra. It can be thought of as a face-centred cubic lattice with a four site basis: $\mathbf{e}_{1}=(-1,-1,-1) / \sqrt{3}, \mathbf{e}_{2}=(1,-1,1) / \sqrt{3}$, $\mathbf{e}_{3}=(1,1,-1) / \sqrt{3}, \mathbf{e}_{4}=(-1,1,1) / \sqrt{3} ; i$ is a unit cell index, and $\alpha$ is the sublattice index.

The dipolar spin ice model contains two terms. First, a nearest-neighbour exchange, $\mathcal{J}$, of strength $J$, to which we add a constant so that its ground states have zero energy; these ground states obey the ice rules or equivalently the constraint that the total pseudospin of each tetrahedron vanish. And second, the dipole interaction $\mathcal{D}$, which is summed over all pairs of sites, with $D=\mu_{0} \mu^{2} /\left(4 \pi r_{\mathrm{nn}}^{3}\right), \mu$ is the magnetic moment of the spins, $r_{n n}$ is the nearest-neighbor distance, and $\mathbf{r}_{i \alpha j \beta}$ is the vector separating spins $\mathbf{S}_{i \alpha}$ and $\mathbf{S}_{j \beta}$. Defining $\mathcal{H} \equiv \sum_{i \alpha, j \beta} \mathcal{H}_{i \alpha, j \beta} \sigma_{i \alpha} \sigma_{j \beta}$, the Hamiltonian is:

$$
\begin{aligned}
\mathcal{H} & =\sum_{\text {pairs }} \sigma_{i \alpha}\left[J \mathcal{J}_{i \alpha, j \beta}+D r_{\mathrm{nn}}^{3} \mathcal{D}_{i \alpha, j \beta}\right] \sigma_{j \beta}, \\
\mathcal{D}_{i \alpha, j \beta} & =\frac{\mathbf{e}_{\alpha} \cdot \mathbf{e}_{\beta}}{\left|\mathbf{r}_{i \alpha, j \beta}\right|^{3}}-\frac{3\left(\mathbf{e}_{\alpha} \cdot \mathbf{r}_{i \alpha, j \beta}\right)\left(\mathbf{e}_{\beta} \cdot \mathbf{r}_{i \alpha, j \beta}\right)}{\left|\mathbf{r}_{i \alpha, j \beta}\right|^{5}}
\end{aligned}
$$

Spectrum and eigenvectors of $\mathcal{H}$ : In this section, we establish the connection of the ice rules with the dipole interaction; this we do by discussing the spectrum and eigenvectors of the Hamiltonian matrix $\mathcal{H}$. These, specifically two dispersionless bands, will form the basis of our discussion of the zero temperature entropy and zero and finite temperature correlations below.

First, consider the spectrum of the adjacency matrix $\mathcal{J}_{i \alpha, j \beta}$. It is well know that this has a pair of degenerate flat bands and two dispersive bands (one of them is gapless at zero wavevector), see Fig. [1] The diagonalizing transformation is given in Ref. 12. We can write the result as the schematic decomposition,

$$
\mathcal{J}=\sum_{\mu=1}^{4} \epsilon_{\mu}(\mathbf{q})\left|v_{\mu}(\mathbf{q})\right\rangle\left\langle v_{\mu}(\mathbf{q})\right|
$$

where for $\mu=1,2, \epsilon_{\mu}(\mathbf{q}) \equiv 0$.

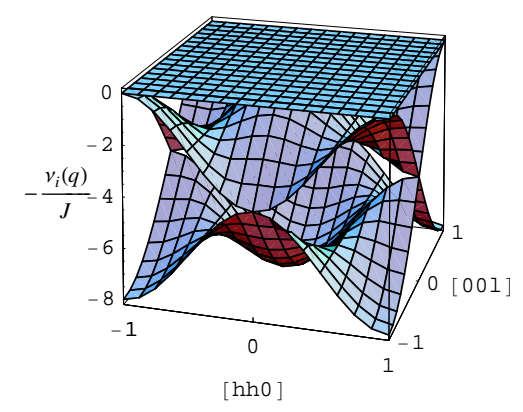

FIG. 1: (color online). Mode spectrum of the nearestneighbor interaction matrix, $\mathcal{J}$, in the $[h h l]$ plane [16], $\mathbf{q}$ is in units of $2 \pi$. Note that here and on the following plots we plot the eigenvalues, $\nu_{i}$, with minus sign.

As shown below, at $T=0$, the physics is determined solely by the modes in the zero-energy flat bands; it will therefore be unchanged for a family of interaction matrices with any other choice of $\epsilon_{3,4}>0$ (but keeping the vectors $v$ unchanged). The family member with $\epsilon_{3,4} \equiv 1$ is then a simple projector $\mathcal{P}=\mathcal{P}^{2}=\sum_{\mu=3}^{4}\left|v_{\mu}(\mathbf{q})\right\rangle\left\langle v_{\mu}(\mathbf{q})\right|$ (Fig. 2).

This is a longitudinal projector; for formal details, see Refs. 12, 13, 14, 15, but in a nutshell, what happens is this. The ice rules $\sum_{i \in t e t} \sigma_{i}$ are equivalent to $\nabla \cdot \mathbf{S}=0$. Here, the spins are thought of link variables ('lattice fluxes') on the diamond lattice, which is dual to the pyrochlore, and $\nabla \cdot$ is the appropriate lattice divergence. This equation encodes the statement that longitudinal modes, those with $\nabla \cdot \mathbf{S} \neq 0$, cost energy, whereas transverse ones do not. The energetically enforced constraint $\nabla \cdot \mathbf{S}=0$ is at the origin of the 'emergent gauge structure' of spin ice, as it can be resolved by transforming to a gauge field (vector potential), $\mathbf{S}=\nabla \times \mathbf{A}$.

What are the matrix elements of $\mathcal{P}$ in real space? Working backwards from the form of the spin ice correlations obtained in [12, 13, 14, 15], one can read off that, asymptotically at large distances, $\mathcal{D} \propto \mathcal{P}$. More precisly, defining a correction term $\Delta$ through

$$
\mathcal{D}_{i \alpha, j \beta}=\frac{8 \pi}{3} \mathcal{P}_{i \alpha, j \beta}+\Delta_{i \alpha, j \beta},
$$

analyticity and symmetry considerations give $\Delta_{i \alpha, j \beta} \sim$ $O\left(r_{i \alpha j \beta}^{-5}\right)$.

Thus, an interaction $\mathcal{P}$ (i) has the same long-distance form as $\mathcal{D}$ and (ii) has the same eigenvectors and same ground-state manifold as $\mathcal{J}$. With this in hand, we can now understand all the important qualitative features of the spectrum of $\mathcal{H}$ analytically and gain a quantitative understanding with computations that do not require us explicitly to treat the conditional convergence of dipole sums. (Note that we again add an overall constant to the energy so that the flat bands occur at zero energy). 
Starting with the model dipole interaction $\mathcal{P}$ (Fig. 2 top panel), it is trivial to add in the superexchange as $\mathcal{J}$ and $\mathcal{P}$ have the same eigenvectors. The same is true of the nearest-neighbor pieces of $\Delta_{i \alpha, j \beta}$, by far its largest matrix elements, which are also proportional to $\mathcal{J}$. The net result is that the lower pair of flat bands remain flat while the upper pair acquire the same dispersion as $\mathcal{J}$ this is illustrated in the middle panel of Fig. 2

Thus far we have shown that "much" of $\mathcal{H}$ is characterized by a pair of low lying flat bands. What remain, the matrix elements of $\Delta_{i \alpha, j \beta}$ beyond the nearest-neighbor distance, are small. Thus their inclusion does not modify the gross features significantly, preserving a spectrum similar to that of the nearest-neighbor model. Indeed, adding $\Delta_{i j}^{\alpha \beta}$ beyond the nearest-neighbor distance weakly splits the remaining pair of flat bands, which acquire a small dispersion; the other pair of bands is barely modified on the scale of their dispersion; this is shown in the figure's bottom panel.

The full $\Delta$ was included by adding its numerical Fourier transform to that of $\mathcal{J}$ and $\mathcal{P}$ obtained analytically, and diagonalising the resulting $4 \times 4$ matrix. With $\Delta_{i \alpha, j \beta}$ small and decaying fast, its Fourier transform is quickly and absolutely convergent; we have checked that the results are essentially independent of the truncation distance for $r_{c} \geq 12 r_{n n}$. We would like to stress that this truncation is not equivalent to the truncation of the long-range dipole interaction since in our case the main long distance part of the dipole interaction is already contained in $\mathcal{P}$.

In Fig. 3. we show the band of maximal eigenvalues of the dipole matrix. It is weakly dispersive because $\Delta_{i j}^{\alpha \beta}$ is small. Our results are in quantitative agreement with the Ewald summation results 11].

Ground states and entropy: The nearest neighbor interaction by itself gives rise to a macroscopic entropy at $T=0$. We now show that any combination of the nearest neighbour, $\mathcal{J}$ and the model dipole, $\mathcal{P}$, interactions leads to precisely the same ground states, and hence entropy.

First, note that the ground states of $\mathcal{J}$ have zero energy; an arbitrary ground state, $|0\rangle$ can thus be written as a linear combination of modes in the flat zero-energy bands only, as an admixture of other bands would lead to a non-zero energy:

$$
|0\rangle=\sum_{\mathbf{q}} \sum_{\mu=1}^{2} a_{\mu}(\mathbf{q})\left|v_{\mu}(\mathbf{q})\right\rangle .
$$

The hard-spin condition imposes constraints on the amplitudes $a_{\mu}(\mathbf{q})$, which we do not need to resolve explicitly.

It suffices to note that, having written the ground states of $\mathcal{J}$ in terms of the flat bands, it follows that they remain ground states upon admixing the model dipole interaction, $\mathcal{P}$, as this only affects the relative position of the excluded positive energy bands $\mu=3,4$. Indeed,
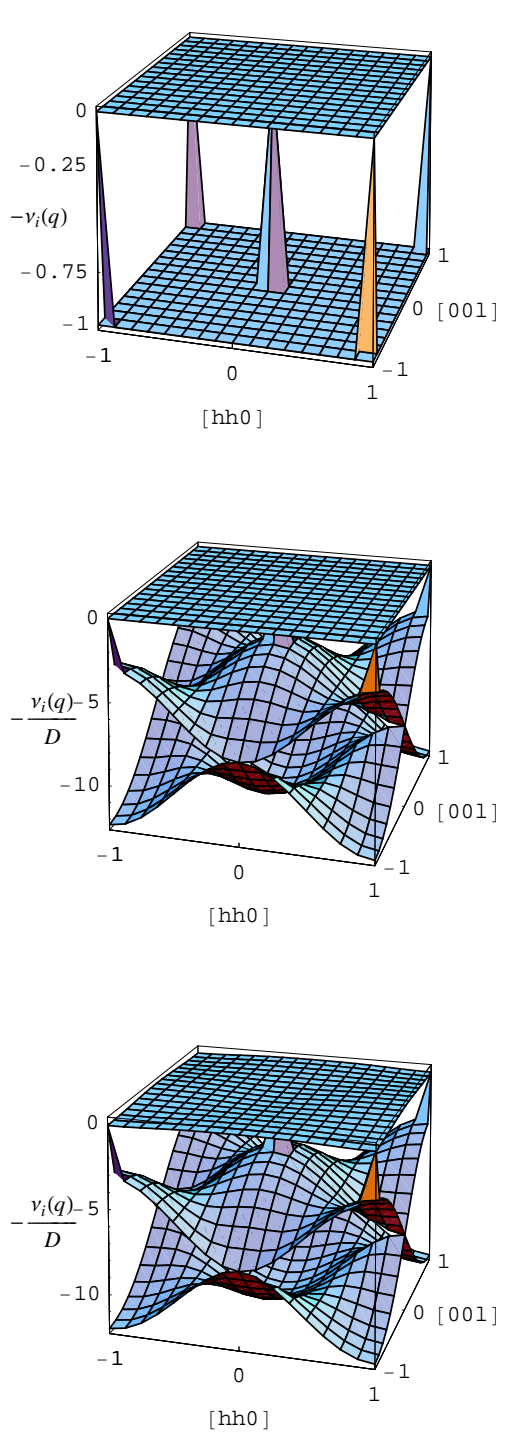

FIG. 2: (color online). Mode spectrum of the dipole interaction (the eigenvalues, $\nu_{i}$, of the $D r_{\mathrm{nn}}^{3} \mathcal{D}_{i j}^{\alpha \beta}$ matrix). Top: Model dipole interaction, $\mathcal{P}$ only. There are two degenerate pairs of flat bands. Middle: After inclusion of nearestneighbor correction. Two bands remain degenerate and flat. The other two become dispersive. Adding a nearest-neighbor superexchange can enhance/suppress this change. Bottom: Correlation function plus $\Delta_{i j}^{\alpha \beta}$. The remaining flat bands become weakly dispersive. The middle and bottom plots are almost identical on this scale. (The spikes in the figure have to with the special character of the single point at $\mathbf{q}=0$ for the conditionally convergent dipole interaction.)

this implies that the set of ground states of $\mathcal{J}$ and $\mathcal{P}$ are identical.

The addition of $\Delta$ will lift this degeneracy but, as we saw, only weakly. Consequently the low temperature entropy of spin ice will be quite close to that of ice before it goes away at the lowest temperatures as the system 


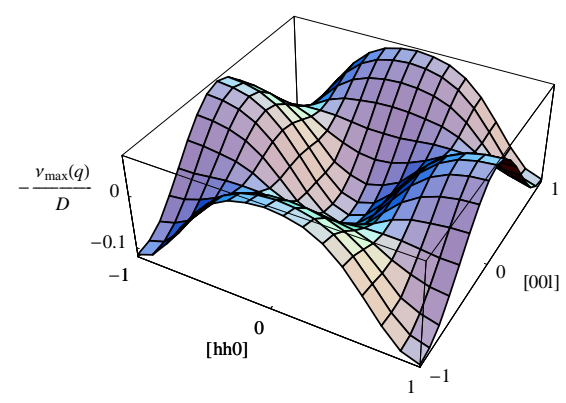

FIG. 3: (color online). Maximum eigenvalue, $\nu_{\max }$, of the dipole matrix in the $[h h l]$ plane, $\mathbf{q}$ is in units of $2 \pi, r_{c}=16 r_{\mathrm{nn}}$.

orders 9 .

Correlations: This explains why the similarity of the spectrum is sufficient to yield the correct lowtemperature physics: for the model dipole interaction, the ground state correlations are exactly those averaged over the ice rule manifold. However, this equivalence between the model dipole and the nearest neighbor antiferromagnetic Ising model breaks down at nonzero temperatures. For the nearest-neighbor Ising model, the presence of thermally activated ice rule violating defects leads to an exponential decay of correlations on a length scale diverging as $\xi \sim \exp (2 J / 3 T)$ at low $T$.

By contrast, for the dipole problem the long range of the interaction implies long ranged correlations at any temperature. This is already evidenced by the first term in the high-temperature expansion of

$$
\left\langle\mathbf{S}_{i \alpha} \cdot \mathbf{S}_{j, \beta}\right\rangle \propto-\mathcal{H}_{i \alpha, j \beta} / T \sim O\left(r_{i \alpha, j \beta}^{-3}\right) .
$$

In fact, in a saddle-point treatment for $\mathcal{P}$, one can show that this holds for any temperature, and to all orders in corrections to the saddle point.

Projective equivalence: The mathematics underlying our analysis of dipolar spin ice can be generalized. One can construct other exchange matrices $\mathcal{J}^{\prime}$ which share their low-lying flat bands (and its eigenvectors) with interactions $\mathcal{D}^{\prime}$ of longer range; we should note though, that generically, neither the $\mathcal{J}^{\prime}$ nor the $\mathcal{D}^{\prime}$ are of bounded range. In this fashion, we find pairs of interactions which are equivalent under projection to the flat bands; this we term "projectively equivalent".

The miracle of spin ice is hence twofold: first, that the physical dipole interaction restricted to the site dependent easy axes on the pyrochlore lattice provides one member of such a pair; and second, thanks to its emergent gauge structure, the other member of the pair is the classic ice problem dating back to Bernal, Fowler and Pauling. In short: dipolar spins are ice because ice is dipolar.

Acknowledgements: We would like to thank Matt Enjalran and Michel Gingras for generously sharing their partially published Ewald summation results with us. We are also grateful to David Huse, Karol Gregor and Werner Krauth for collaboration on closely related work. We thank the above, Chris Henley, and Oleg Tchernyshyov for useful discussions. This work was in part supported by the Ministère de la Recherche et des Nouvelles Technologies, by the NSF [PHY99-07949 (at KITP), 0213706] and by the David and Lucile Packard Foundation.

[1] P. W. Anderson, Phys. Rev. 102, 1008 (1956).

[2] L. Pauling, J. Am. Chem. Soc. 57, 2680 (1935); L. Pauling, The Nature of the Chemical Bond, (Cornell University Press, Ithaca, NY, 1965).

[3] M. J. Harris, S. T. Bramwell, D. F. McMorrow, T. Zeiske, and K. W. Godfrey, Phys. Rev. Lett. 79, 2554 (1997).

[4] A. P. Ramirez, A. Hayashi, R. J. Cava, R. Siddharthan, and B. S. Shastry, Nature 399, 333 (1999).

[5] R. Siddharthan, B. S. Shastry, A. P. Ramirez, A. Hayashi, R. J. Cava, and S. Rosenkranz, Phys. Rev. Lett. 83, 1854 (1999); S. Rosenkranz, A. P. Ramirez, A. Hayashi, R. J. Cava, R. Siddharthan, and B. S. Shastry, J. Appl. Phys. 87, 5014 (2000); R. Siddharthan, B. S. Shastry, and A. P. Ramirez, Phys. Rev. B 63, 184412 (2001); B. S. Shastry, Physica B 329-333, 1024 (2003).

[6] B. C. den Hertog and M. J. P. Gingras, Phys. Rev. Lett. 84, 3430 (2000).

[7] S. T. Bramwell, M. J. Harris, B. C. den Hertog, M. J. P. Gingras, J. S. Gardner, D. F. McMorrow, A. R. Wildes, A. L. Cornelius, J. D. M. Champion, R. G. Melko, and T. Fennell, Phys. Rev. Lett. 87, 047205 (2001).

[8] R.G. Melko, B. C. den Hertog, and M. J. P. Gingras, Phys. Rev. Lett. 87, 067203 (2001).

[9] The predicted ordering has never been experimentally observed, apparently because it occurs at a temperature where the system is unable to reach its equilibrium state [J. Snyder, J. S. Slusky, R. J. Cava, and P. Schiffer, Nature 413, 48 (2001); K. Matsuhira, Y. Hinatsu, and T. Sakakibara, J. Phys.: Cond. Mat. 13, L737 (2001)].

[10] M. J. P. Gingras and B. C. den Hertog, Can. J. Phys. 79, 1339 (2001).

[11] M. Enjalran and M. J. P. Gingras, Phys. Rev. B 70, 174426 (2004); R.G. Melko and M. J. P. Gingras, J. Phys.: Cond. Mat. 16, R1277 (2004).

[12] S. V. Isakov, K. Gregor, R. Moessner, and S. L. Sondhi, Phys. Rev. Lett. 93, 167204 (2004).

[13] D. A. Huse, W. Krauth, R. Moessner, and S. L. Sondhi, Phys. Rev. Lett. 91, 167004 (2003); R. Moessner and S. L. Sondhi, Phys. Rev. B 68, 184512 (2003).

[14] M. Hermele, M. P. A. Fisher, and L. Balents, Phys. Rev. B 69, 064404 (2004).

[15] C. L. Henley, Phys. Rev. B 71, 014424 (2005).

[16] D. A. Garanin and B. Canals, Phys. Rev. B 59, 443 (1999); B. Canals and D. A. Garanin, Can. J. Phys. 79, 1323 (2001).

[17] Our treatment follows the introduction to large- $N$ by M. Moshe and J. Zinn-Justin, Phys. Rep. 385, 69 (2003). 